\title{
Peer-to-Peer File Sharing Game using Correlated Equilibrium
}

\author{
Beibei Wang, Zhu Han*, and K. J. Ray Liu \\ Department of Electrical and Computer Engineering and Institute for Systems Research, \\ University of Maryland, College Park, MD 20742, USA \\ * Department of Electrical and Computer Engineering, University of Houston, Houston, TX 77004, USA
}

\begin{abstract}
Peer-to-peer (P2P) systems have become more and more popular in nowadays by providing decentralized, selforganizing and fault tolerant file sharing services. As the selfish users do not benefit from providing free service, they tend to download files from other users and yet not to upload for the others, resulting in low system efficiency. In this paper, we propose a correlated equilibrium-based file sharing game to enhance users' performance. We first characterize users' utility with their expected delay. Then, using the correlated equilibrium concept, instead of optimizing their own benefits alone, the users are aware of the best response for them to jointly optimize their strategies together and achieve the correlated equilibrium. Simulation results are presented to demonstrate the efficiency of the proposed scheme.
\end{abstract}

\section{INTRODUCTION}

With the rapid development of networking and communication technologies, in recent years, peer-to-peer (P2P) file sharing services have become more and more popular, such as Napster [1], Gnutella [2], KaZaA [3], and BitTorrent [4]. By combining sophisticated searching techniques with a large scale decentralized file storage, $\mathrm{P} 2 \mathrm{P}$ file sharing systems allow users to download files directly from each other. Therefore, they offer a decentralized, self-organizing, scalable, and fault tolerant file sharing service providing an effective balancing of storage and bandwidth resources [5].

Current P2P systems rely on the assumption that users will altruistically serve files to the community for free. However, reserving a certain upload bandwidth to serve the others incurs a cost to a user. Since users are selfish and do not benefit from providing free service, many of them tend not to contribute to uploading their files while leech the other users [6]. Hence, it is of great importance to motivate user cooperation in $\mathrm{P} 2 \mathrm{P}$ systems to make more files available. In the literature, various incentive schemes based on game-theoretic modeling have been proposed to increase the proportion of users that share files. Tit-for-tat strategy was proposed in BitTorrent application to prevent user free-riding [7]-[9]. The authors of [10] studied the incentives for cooperation based on repetition and reputation by modeling file sharing as an evolutionary prisoner's dilemma. A differential servicebased incentive scheme was proposed in [11] to improve the system's performance. The work in [12] proposed a micropayment incentive mechanism in which users earn rewards by uploading to others and pay for downloading. The authors of [13] modeled the bandwidth allocation in P2P systems as an exchange economy and proposed the proportional response dynamics to achieve the market equilibrium.
However, most of the existing game-theoretic frameworks of P2P systems assumed that the system users optimize their own benefits individually without considering the other users' actions, and would stick to noncooperative strategies even at the high risk of having no user upload files and suffering poor individual performance. In this paper, we propose a correlated equilibrium-based file sharing game to enhance the users' performance. We first model the file sharing as a non-cooperative game, where we characterize the users' utility using the expected delay they experience in file sharing. Specifically, if no users contribute to uploading files for the others, the server has to respond to all data requests from the users and the users may experience a long delay. If some of the users cooperate in uploading files, all the other users can download files from both the server and the cooperative users, so the expected delay can be reduced. The noncooperative users who free ride may enjoy a shorter delay, but are also aware of the risk of having an unaffordable delay if no one cooperates. Using the correlated equilibrium (CE) concept [15], the selfish but rational users are aware of the risk of having no one contribute to uploading files, so that it is the best response for them to jointly optimize their actions and achieve a higher degree of cooperation at $\mathrm{CE}$ with a better performance. Simulation results also illustrates that the expected delay at $\mathrm{CE}$ is greatly reduced compared to the delay performance at Nash equilibrium.

The rest of the paper is organized as follows. In Section II, we present the system model of P2P file sharing and derive the expected delay using queuing theory. We model file sharing as a noncooperative game in Section III, and analyze the proposed game using the correlated equilibrium concept in Section IV. Simulation results are presented in Section V. Finally, Section VI concludes the paper.

We adopt the following notation: $\lambda$ and $\mu$ are arrival rate and service rate, respectively. $D$ is the delay, $R$ is the download rate, $r$ is the upload rate, $U$ is the utility, $p$ is the probability, $E$ is the expectation, $S$ is user set, $\Omega$ is the action set, and $\mathbb{R}$ is the regret.

\section{SySTEM MODEL}

In this section, we define a general model of a P2P file sharing system in which we assume symmetric exchange of data between peers. By symmetric, we mean that the server has all the required data of all peers, while some big data file is divided into several chunks, each peer owns some of the chunks, and can download the remaining chunks from 
either the server or the other peers who would contribute some bandwidth for uploading data. We begin by modelling a simple two-peer $(N=2)$ file sharing, and then generalize it to multiple peers $(N>2)$.

\section{A. Two-Peer File Sharing}

In general, the server has only a limited bandwidth, so not all the requests for data chunks from the peers can be immediately satisfied, and the peers may experience delay. Thus, in this paper, we characterize the performance in file sharing by the expected delay a peer experiences (i.e., mean steady-state time in queue).

Without loss of generality, we assume for the arrival process of the chunk requests, the inter-arrival time is exponentially distributed with parameter $\lambda^{-1}$, and the duration that a data source (i.e., the server or a peer) uploads a chunk is $\mu^{-1}$. Then we can model the file service process as an $\mathrm{M} / \mathrm{D} / 1$ queue, and the delay function, denoted by $f(\lambda, \mu)$, can be written as [21]

$$
f(\lambda ; \mu)=\frac{\lambda}{2 \mu(\mu-\lambda)} .
$$

In Figure 1, we show the system model for two-peer file sharing. For case I, neither peer 1 nor peer 2 would upload data chunks for each other, so they both send their requests to the server, and the expected delay for peer 1 and peer 2 in this case can be represented as

$$
D_{i}=f\left(R_{1}+R_{2} ; r_{0}\right), i=1,2,
$$

where $R_{1}$ and $R_{2}$ is the downloading rate (or chunk request rate) of peer 1 and peer 2 , respectively, and $r_{0}$ is the uploading rate (or service rate, uploading bandwidth) of the server, with stability requirement $R_{1}+R_{2}<r_{0}{ }^{1}$.

As we mentioned earlier, both the peers may experience a certain delay due to the limited capacity/bandwidth of the server. A possibly better choice for the peers is to contribute some bandwidth to uploading data so that they can download chunks from two sources, which may potentially lower the burden of the server and reduce the delay. This corresponds to case II in Figure 1 where both peer 1 and peer 2 cooperate. We assume that each peer requests data chunks from the server and the other peer with equal probability, so the downloading rate from either of them is $R_{i} / 2$. We also assume the total upload bandwidth $r$ is equally shared by the two cooperative peers. Since the queuing process at the server and each peer is assumed to be independent from each other, by applying Little's Law [21], we get the expected delay of peer $i$ as

$$
D_{i}=\frac{1}{2}\left[f\left(\frac{R_{1}+R_{2}}{2} ; r_{0}\right)+f\left(\frac{R_{i}}{2} ; \frac{r}{2}\right)\right], i=1,2,
$$

with stability requirement $R_{1}+R_{2}<2 r_{0}$ and $R_{i}<r$.

However, peers in $\mathrm{p} 2 \mathrm{p}$ file sharing are selfish and would prefer to download chunks from the other peers rather than contribute to upload for the others, since reserving uploading

\footnotetext{
${ }^{1}$ We assume throughout the paper that the stability requirements are always satisfied when using (1), otherwise we set the delay as infinity without using (1).
}

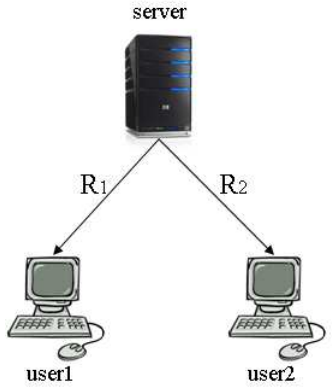

I. both peers non-cooperate

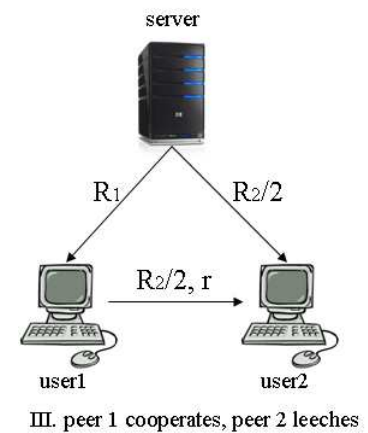

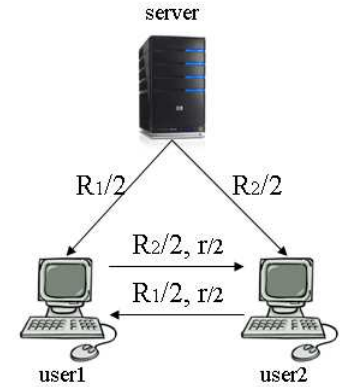

II. both peers cooperate

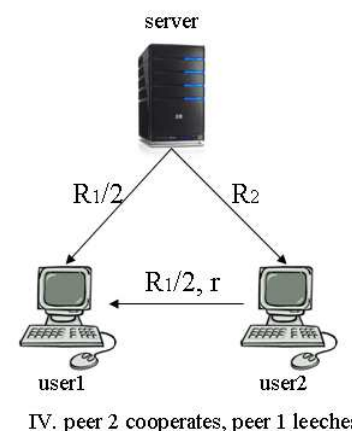

Fig. 1. System model

bandwidth incurs cost to peers. This case is illustrated in case III and IV of Figure 1. In case III, where peer 1 is cooperative and peer 2 leeches, we have the peer's expected delay as

$$
D_{1}=f\left(R_{1}+\frac{R_{2}}{2} ; r_{0}\right),
$$

and

$$
D_{2}=\frac{1}{2}\left[f\left(R_{1}+\frac{R_{2}}{2} ; r_{0}\right)+f\left(\frac{R_{2}}{2}, r\right)\right],
$$

with stability requirement $2 R_{1}+R_{2}<2 r_{0}$ and $R_{2}<2 r$.

Similarly for case IV where peer 1 leeches and peer 2 is cooperative, we have the expected delay as

$$
D_{1}=\frac{1}{2}\left[f\left(\frac{R_{1}}{2}+R_{2} ; r_{0}\right)+f\left(\frac{R_{1}}{2}, r\right)\right],
$$

and

$$
D_{2}=f\left(\frac{R_{1}}{2}+R_{2} ; r_{0}\right),
$$

with stability requirement $R_{1}+2 R_{2}<2 r_{0}$ and $R_{1}<2 r$.

\section{B. $N$-Peer File Sharing $(N>2)$}

In the following, we extend the derivation above to $\mathrm{N}$-peer file sharing with $N>2$. Assume there are in total $N$ peers, denoted by set $\mathcal{S}$. A subset $\mathcal{S}_{c} \subseteq \mathcal{S}$, would reserve their own bandwidth for uploading data chunks. Then the remaining noncooperative peers form a set $\mathcal{S}_{n}=\mathcal{S} \backslash \mathcal{S}_{c}$.

We further assume a peer directs his/her requests to the server and all the cooperative peers with equal probability 
for simplicity. Hence, if peer $i$ chooses to cooperate (C) in uploading data, he/she can either download data from the server or the remaining $(K-1)$ other cooperative peers, and the request rate of peer $i$ to each of them is essentially $\frac{R_{i}}{(1-K)+1}=\frac{R_{i}}{K}$. On the other hand, peer $j$ who prefers not to cooperate $(\mathrm{N})$ can choose downloading from the server or the $K$ cooperative peers, and his/her request rate to each data provider is $\frac{R_{j}}{K+1}$. Therefore, for the queue at the server side, the arrival rate of data requests is

$$
\lambda_{s}=\sum_{j \in \mathcal{S}_{n}} \frac{R_{j}}{K+1}+\sum_{i \in \mathcal{S}_{c}} \frac{R_{i}}{K},
$$

and the service rate is $\mu_{s}=r_{0}$.

For the queue at a cooperative peer, say peer $i$, the arrival rate of requests is

$$
\lambda_{p_{i}}=\sum_{j \in \mathcal{S}_{n}} \frac{R_{j}}{K+1}+\sum_{l \in \mathcal{S}_{c}, l \neq i} \frac{R_{l}}{K},
$$

and the service rate is $\mu_{p_{i}}=\frac{r}{K}$ since the $K$ cooperative peers equally contribute to an upload bandwidth of $r$.

Therefore, from the assumption that each queue is independent and using Little's Law [21], we obtain the expected delay of a cooperative peer $i$ as

$$
D_{p_{i}}^{c}=\frac{1}{K}\left[f\left(\lambda_{s} ; \mu_{s}\right)+\sum_{l \in \mathcal{S}_{c}, l \neq i} f\left(\lambda_{p_{l}} ; \mu_{p_{l}}\right)\right] \text {, if } K \in[1, N],
$$

and the delay of a non-cooperative peer $j$ is

$$
D_{p_{j}}^{n}=\frac{1}{K+1}\left[f\left(\lambda_{s} ; \mu_{s}\right)+\sum_{i \in \mathcal{S}_{c}} f\left(\lambda_{p_{i}} ; \mu_{p_{i}}\right)\right] \text {, if } K \in[1, N-1] \text {, }
$$

and

$$
D_{p_{j}}^{n}=f\left(\sum_{i \in \mathcal{S}} R_{i} ; \mu_{s}\right), \text { if } K=0 .
$$

\section{File Sharing Game ANd Correlated EQUILIBRIUM}

As the peers are greedy, and aims at minimizing their delay, we can model the file sharing as a game. Let $\mathbb{G}=$ $\left\{\mathcal{S},\left(\Omega_{i}\right)_{i \in \mathcal{S}},\left(U_{i}\right)_{i \in \mathcal{S}}\right\}$ be a finite $N$-player ${ }^{2}$ game in strategic form, where $\Omega_{i}=\{C, N\}$ (C stands for cooperation and $N$ stands for non-cooperation) is the strategy space for player $i$, and $U_{i}$ is the utility function for player $i$. Define $\Omega_{-i}$ as the strategy space for player $i$ 's opponents, and denote the action for player $i$ and his/her opponents as $r_{i}$ and $r_{-i}$, respectively. In the file sharing game, since the peers try to minimize their expected delay, we can define their utility function as a nonincreasing function of the expected delay. For simplicity, we use the negative of the expected delay a peer experiences as his/her utility, i.e., $U_{i}=-D_{i}$. In other word, we want to maximize the utility by minimize the delay. Other utility functions with similar properties can also be analyzed, e.g. the delayed sensitive utilities for multimedia applications.

\footnotetext{
${ }^{2}$ We use user, player and peer interchangeably.
}

Nash equilibrium (NE) is a well-known concept to characterize the outcome of a game, which states that at equilibrium every player will select a utility-maximizing strategy given the strategies of every other player. However, computing the mixed-strategy $\mathrm{NE}$ of a $N$-player game in polynomial time is generally very difficult, since it requires to solve multiple highorder polynomial equations. Nevertheless, correlated equilibrium can be computed in polynomial time in essentially all kinds of multi-player games [20]. Further, due to peers' selfish nature, NE may not be system efficient as peers only aim at maximizing their individual utility (i.e., minimizing the delay). Therefore, we next study the concept of correlated equilibrium (CE), which is more general than the NE [15].

The idea of CE is that a strategy profile is chosen randomly according to a certain distribution. Define $p\left(r_{i}, r_{-i}\right)$ as the joint distribution of users to perform a specific action. Given the recommended strategy, it is to the players' best interests to conform with this strategy, and the distribution is called the correlated equilibrium, i.e., for all $i \in \mathcal{S}, r_{i}, r_{i}^{\prime} \in \Omega_{i}$, and $r_{-i} \in \Omega_{-i}$,

$$
\sum_{r_{-i} \in \Omega_{-i}} p\left(r_{i}, r_{-i}\right)\left[U_{i}\left(r_{i}^{\prime}, r_{-i}\right)-U_{i}\left(r_{i}, r_{-i}\right)\right] \leq 0 .
$$

Inequality (13) means that when the recommendation to player $i$ is to choose action $r_{i}$, then choosing action $r_{i}^{\prime}$ instead of $r_{i}$ cannot result in a higher expected utility to player $i$. In the file sharing game, peers can obtain some information from the server, such as data request rates of other peers, the total shared bandwidth $(r)$, and the server's uploading rate. This way, they will consider the potential long delay when no user contributes to serving files, and it is the best response for them to jointly optimize their actions so as to attain a higher degree of cooperation and reduce delay.

We note from (13) that the set of correlated equilibria is nonempty, closed and convex in every finite game [16]. Moreover, it may include the distribution that is not in the convex hull of the Nash equilibria. In fact, every Nash equilibrium is a point inside the correlated equilibria set, and the $\mathrm{NE}$ corresponds to the special case where $p\left(r_{i}, r_{-i}\right)$ is a product of each individual user's probability for different actions, i.e., the action of the different players is independent [15], [16].

Among the multiple $\mathrm{CE}$, which one is the most suitable should be very carefully considered in practical design. In [17] [22], the authors proposed the criterion of correlated optimal, which is the solution that achieves the highest social welfare, i.e.,

Definition 1: A multi-strategy $\mathbf{r}^{\text {all }}$ is correlated optimal if it satisfies the following conditions,

$$
\begin{gathered}
p\left(\mathbf{r}^{\text {all }}\right)=\arg \max _{p} \sum_{i \in \mathcal{S}} E_{p}\left(U_{i}\right), \\
\text { s.t. } \quad \sum_{r_{-i} \in \Omega_{-i}} p\left(r_{i}, r_{-i}\right)\left[U_{i}\left(r_{i}^{\prime}, r_{-i}\right)-U_{i}\left(r_{i}, r_{-i}\right)\right] \leq 0,
\end{gathered}
$$

$\forall r_{i}, r_{i}^{\prime} \in \Omega_{i}$, and $\forall i \in \mathcal{S}$.

Other criterion can also be considered, such as the max-min 
fairness criterion, defined as

$$
p\left(\mathbf{r}^{\text {fair }}\right)=\arg \max _{p} \min E_{p}\left(U_{i}\right) .
$$

TABLE I

TWO-PEER GAME (A) UTILITY TABLE (LEFT); (B) STRATEGY NOTATION (RIGHT).

\begin{tabular}{|c|c|c|}
\hline (a) & $C$ & $N$ \\
\hline$C$ & $\left(U_{1,1}^{(1)}, U_{1,1}^{(2)}\right)$ & $\left(U_{1,2}^{(1)}, U_{1,2}^{(2)}\right)$ \\
\hline$N$ & $\left(U_{2,1}^{(1)}, U_{2,1}^{(2)}\right)$ & $\left(U_{2,2}^{(1)}, U_{2,2}^{(2)}\right)$ \\
\hline
\end{tabular}

\begin{tabular}{|c|c|c|}
\hline (b) & $C$ & $N$ \\
\hline$C$ & $p_{1,1}$ & $p_{1,2}$ \\
\hline$N$ & $p_{2,1}$ & $p_{2,2}$ \\
\hline
\end{tabular}

Overall, the generalized system model is to employ any system optimization goal with the correlated equilibrium constraint and other physical constraints, so as to achieve higher mutual benefits by cooperating on the joint probability distribution of users' actions. In this paper, we use sum utility as an example, but other optimization criteria can be employed in a similar way.

\section{Analysis of the Proposed Game}

In this section, we investigate two approaches for the proposed game. The first one is by linear programming method, and the second one is distributed learning algorithm.

\section{A. Linear Programming Method}

We study the properties of CE using a two-peer game as an example. A general utility table is shown in Table I, in which $U_{i, j}^{(k)}$ is the utility for player $k$ when the joint action pair is in the $i^{t h}$ row and the $j^{\text {th }}$ column, and $p_{i, j}$ is defined as the corresponding joint probability for that action pair.

The linear programming problem (14) to obtain the correlated optimal strategy becomes

$$
\max _{\left\{p_{i, j}\right\}} \sum_{j=1}^{2} \sum_{i=1}^{2}\left(U_{i, j}^{(1)}+U_{i, j}^{(2)}\right) p_{i, j},
$$

constrained by

$$
\begin{aligned}
& p_{1,1}+p_{1,2}+p_{2,1}+p_{2,2}=1, \\
& p_{1,1}\left(U_{1,1}^{(1)}-U_{2,1}^{(1)}\right) \geq p_{1,2}\left(U_{2,2}^{(1)}-U_{1,2}^{(1)}\right), \\
& p_{2,1}\left(U_{2,1}^{(1)}-U_{1,1}^{(1)}\right) \geq p_{2,2}\left(U_{1,2}^{(1)}-U_{2,2}^{(1)}\right), \\
& p_{1,1}\left(U_{1,1}^{(2)}-U_{1,2}^{(2)}\right) \geq p_{2,1}\left(U_{2,2}^{(2)}-U_{2,1}^{(2)}\right), \\
& p_{1,2}\left(U_{1,2}^{(2)}-U_{1,1}^{(2)}\right) \geq p_{2,2}\left(U_{2,1}^{(2)}-U_{2,2}^{(2)}\right),
\end{aligned}
$$

where inequalities (18)-(21) represent the constraints of CE in (13).

Let $\Delta^{3}=\left\{\left(p_{1,1}, \cdots, p_{2,2}\right) \in \mathbf{R}_{+}^{4} \mid p_{1,1}+\cdots+p_{2,2}=1\right\}$ denote the 3-dimensional simplex of $\mathbf{R}^{4}$. Since the above constraints and the objective function are all linear, similar to [18], we can derive the set of correlated equilibria as follows.

Lemma 1: The set of correlated equilibria for the game defined in Table $\mathrm{I}$ is a hexahedron of $\Delta^{3}$ with five vertices
TABLE II

FIVE VERTICES OF THE CORRELATED EQUILIBRIA SET

\begin{tabular}{|c|c|c|c|c|}
\hline$p$ & $p_{1,1}$ & $p_{2,2}$ & $p_{2,1}$ & $p_{1,2}$ \\
\hline NE1 & 0 & 0 & 0 & 1 \\
\hline NE2 & 0 & 0 & 1 & 0 \\
\hline NE3 & $\frac{1}{(1+a)(1+b)}$ & $\frac{a b}{(1+a)(1+b)}$ & $\frac{b}{(1+a)(1+b)}$ & $\frac{a}{(1+a)(1+b)}$ \\
\hline CE1 & $\frac{1}{1+a+b}$ & 0 & $\frac{b}{1+a+b}$ & $\frac{a}{1+a+b}$ \\
\hline CE2 & 0 & $\frac{a b}{a+b+a b}$ & $\frac{b}{a+b+a b}$ & $\frac{a}{a+b+a b}$ \\
\hline
\end{tabular}

given in Table II, where

$$
a=\frac{\left|U_{1,1}^{(1)}-U_{2,1}^{(1)}\right|}{\left|U_{2,2}^{(1)}-U_{1,2}^{(1)}\right|}, \text { and } b=\frac{\left|U_{1,1}^{(2)}-U_{1,2}^{(2)}\right|}{\left|U_{2,2}^{(2)}-U_{2,1}^{(2)}\right|} .
$$

Because (16) is a linear programming problem, its solution will be one of the five vertices shown in Table II, according to the coefficients of $p_{i, j}$. Specifically, NE1 and NE2 are the two pure-strategy NE, and will be the solution to the problem in (16) if $U_{1,2}^{(1)}+U_{1,2}^{(2)}$ (or $U_{2,1}^{(1)}+U_{2,1}^{(2)}$ ) is the largest among all $U_{i, j}^{(1)}+U_{i, j}^{(2)}$ s. However, it is more often the case that the highest social welfare is achieved if all players cooperate, i.e. $U_{1,1}^{(1)}+U_{1,1}^{(2)}$ is the largest coefficient in (16), and when no user cooperates the social welfare $U_{2,2}^{(1)}+U_{2,2}^{(2)}$ is the lowest. Due to the CE constraints, always cooperating can not be achieved among the selfish users $\left(p_{1,1}<1\right.$ in all five vertices). However, since the users who adopts CE strategy now jointly optimize their actions by considering the low utility of mutual non-cooperation and the high utility when both cooperate, the degree of cooperation is increased. For instance, at CE1, $p_{1,1}$ is the largest and $p_{2,2}$ is the smallest among the five vertices, so the social welfare at CE1 is the highest. Moreover, since the set of Nash equilibria is formed by all convex combinations of the three NE vertices, it is impossible to achieve an even higher social welfare in the NE set than that of CE1. Therefore, the system performance using the correlated optimal strategy will be higher than any strategy in the NE set.

For multiuser asymmetric case, the linear programming can still be employed. The solution can be computational easy due to some fast algorithms such as Simplex algorithm [23]. However, extensive signalling might be necessary to gather all the information. Next, we will propose a distributed solution.

\section{B. Multiuser Distributed Learning Algorithm}

It is shown that regret-matching algorithm [16] can converge to the set of CE. Specifically, for any two distinct actions $r_{i} \neq r_{i}^{\prime}$ in $\Omega_{i}$, the regret of user $i$ at time $T$ for not playing $r_{i}^{\prime}$ is

$$
\mathbb{R}_{i}^{T}\left(r_{i}, r_{i}^{\prime}\right):=\max \left\{Q_{i}^{T}\left(r_{i}, r_{i}^{\prime}\right), 0\right\},
$$

where

$$
Q_{i}^{T}\left(r_{i}, r_{i}^{\prime}\right)=\frac{1}{T} \sum_{t \leq T}\left(U_{i}^{t}\left(r_{i}^{\prime}, r_{-i}\right)-U_{i}^{t}\left(r_{i}, r_{-i}\right)\right) .
$$


TABLE III

REGRET-MATCHING LEARNING ALGORITHM

Initialize arbitrarily probability for taking action of user $i$, $p_{i}^{1}\left(r_{i}\right), \forall i \in K$.

For $\mathrm{t}=2,3,4, \ldots$

1. Find $Q_{i}^{t-1}\left(r_{i}, r_{i}^{\prime}\right)$ as in (24).

2. Find average regret $\mathbb{R}_{i}^{t-1}\left(r_{i}, r_{i}^{\prime}\right)$ as in (23).

3. Let $r_{i} \in \Omega_{i}$ be the strategy last chosen by user $i$, i.e. $r_{i}^{t-1}=r_{i}$. Then the probability distribution of the actions for the next period, $p_{i}^{t}$ is defined as

$p_{i}^{t}\left(r_{i}^{\prime}\right)=\frac{1}{\mu} \mathbb{R}_{i}^{t-1}\left(r_{i}, r_{i}^{\prime}\right) \quad \forall r_{i}^{\prime} \neq r_{i}$,

$p_{i}^{t}\left(r_{i}\right)=1-\sum_{r_{i}^{\prime} \neq r_{i}} p_{i}^{t}\left(r_{i}^{\prime}\right)$,

where $\mu$ is a certain constant that is sufficiently large.

$Q_{i}^{T}\left(r_{i}, r_{i}^{\prime}\right)$ can be viewed as the average extra payoff that user $i$ would have obtained, if it had played action $r_{i}^{\prime}$ every time in the past instead of choosing $r_{i}$, and $\mathbb{R}_{i}^{T}\left(r_{i}, r_{i}^{\prime}\right)$ can be viewed as a measure of the average regret. The probability $p_{i}\left(r_{i}\right)$ for user $i$ to take action $r_{i}$ is a linear function of the regret. The details of the regret-matching algorithm is shown in Table III. The complexity of the algorithm is $O\left(\left|\Omega_{i}\right|\right)$.

For every period of $T$, let's define the relative frequency of users' joint action $\mathbf{r}$ played till $T$ periods of time as

$$
z_{T}(\mathbf{r})=\frac{1}{T} \#\left\{t \leq T: \mathbf{r}_{t}=\mathbf{r}\right\}
$$

where $\#(\cdot)$ denotes the number of times the event inside the bracket happens and $\mathbf{r}_{t}$ is all users' action at time $t$. It is proved in [16] that $z_{T}$ converges almost surely to a set of CE in the long run if every player adjusts strategy according to the algorithm in Table III.

However, $z_{T}$ after convergence may not be the optimal correlated strategy. Actually in general, the learning algorithm in Table III will learn the mixed-strategy NE, which is worse than the optimal CE strategy. In order to obtain the optimal CE strategy by maximizing the objective function in (16), we next derive a new learning algorithm, which allows negotiation between players.

\section{Preliminary Simulation Results}

In order to evaluate the delay performance in the proposed file sharing game, we conducted several preliminary simulations. We assume a residential network, where the downloading rate of each user is set to be $R=400 \mathrm{~kb} / \mathrm{s}$, and the service rate (uploading rate) of server is $r_{0}=1250$ $\mathrm{kb} / \mathrm{s}$. We assume three peers are in the P2P system, and vary the shared upload bandwidth $r$ in the interval of $[420,1800]$ $\mathrm{kb} / \mathrm{s}$.

In Figure 2, we plot the probability of peer cooperation at mixed-strategy NE. Since the peers' downloading rates are identical, the file sharing game is symmetric in terms of the utility, so the probability of cooperation is the same for all three peers. It can be seen from Figure 2 that as the shared upload bandwidth increases, the probability of cooperation also increases. This is because when peers share a greater

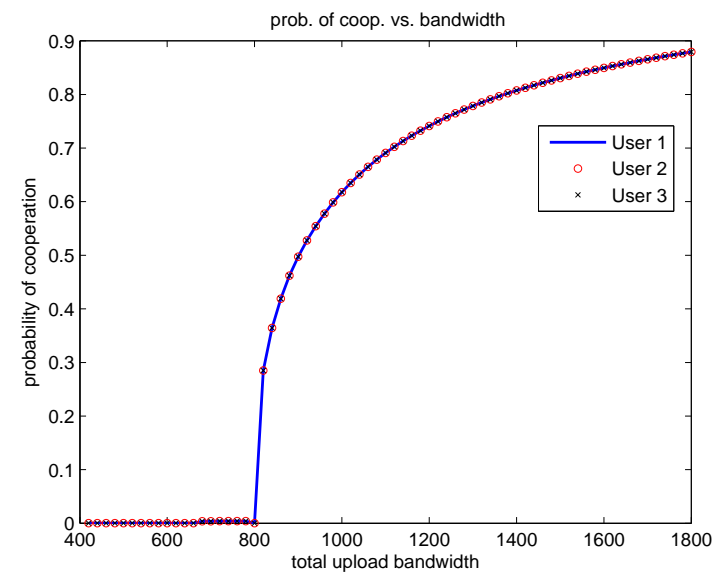

Fig. 2. Probability of cooperation at mixed-strategy NE.

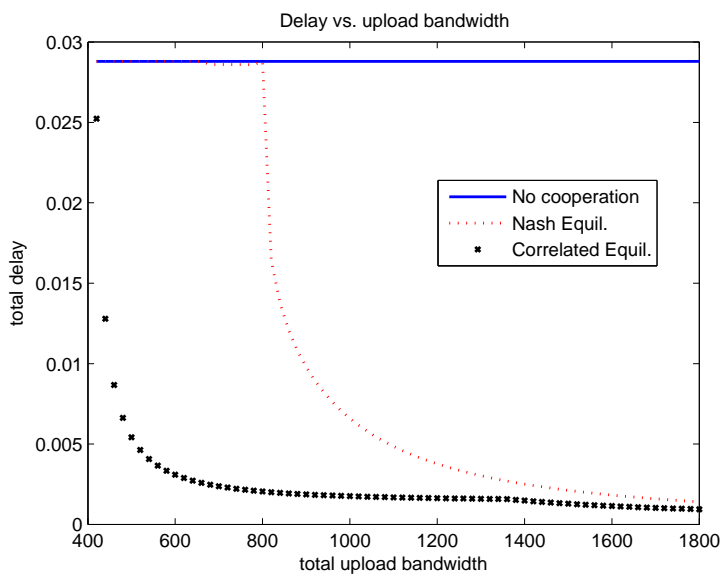

Fig. 3. Comparison of total delay.

upload bandwidth, a higher degree of cooperation can more greatly reduce an individual user's delay.

In Figure 3, we compare the total delay of the three users under no-cooperation, mixed-strategy $\mathrm{NE}$, and the correlated optimal strategy. We see that when there is no cooperation, the users experience a very long delay; since the users only rely on the server to download files, the delay is a horizontal line with $r$ increasing. At the mixed-strategy NE, the delay decreases rapidly as $r$ increases, because the peers share an increasing upload bandwidth and become more cooperative. The CE strategy has the best performance, since the users now jointly optimize their actions. Moreover, as the delay does not decrease too much when $r$ grows beyond $900 \mathrm{~kb} / \mathrm{s}$, it suffices to share a total upload bandwidth at around $900 \mathrm{~kb} / \mathrm{s}$, in order to reduce the cost to the users.

In Figure 4, we show the probability of joint actions of all three users at their correlated optimal strategy. The y-axis represents the probability that a subset of the peers choose cooperation, and the indices of the cooperative peers are listed inside the square brackets beside each subplot. Since the probability of all peers cooperating and the probability 

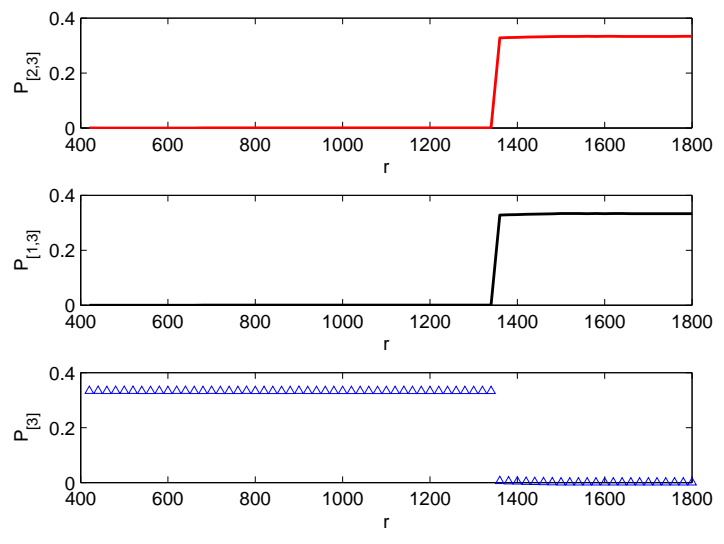

(a) Joint probabilities when peer 3 cooperates
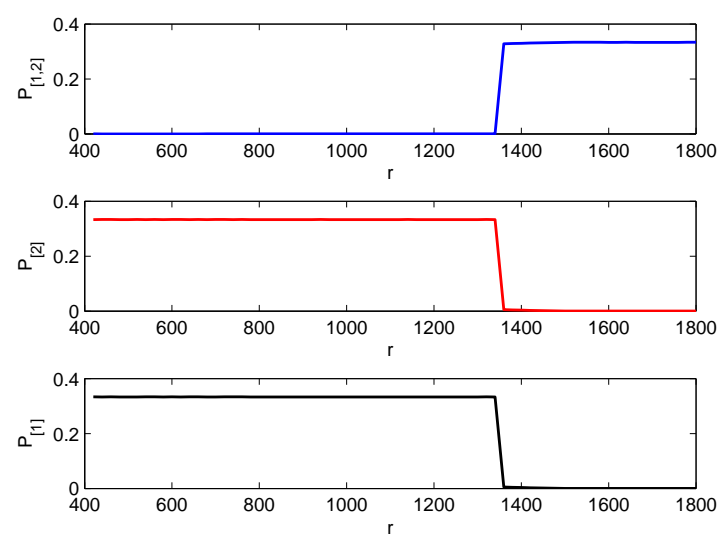

(b) Joint probabilities when peer 3 does not cooperate

Fig. 4. Joint probability at the correlated optimal strategy (the index inside the square brackets indicates which user contributes to uploading).

of no peer cooperating are both very close to zero, we only show the other nonzero probabilities. We can see that when the shared upload bandwidth $r$ is less than $1350 \mathrm{~kb} / \mathrm{s}$, since the probability of only one peer cooperating is around $1 / 3$, the correlated optimal strategy randomly suggests one user to provide service to the other two. The reason why no more users choose cooperation is that the shared bandwidth is relatively small. For instance, if three users share a $900 \mathrm{~kb} / \mathrm{s}$ upload bandwidth, each of them can only offer $300 \mathrm{~kb} / \mathrm{s}$ uploading rate, while their request rates are $400 \mathrm{~kb} / \mathrm{s}$. More users will choose cooperation when the shared bandwidth is greater. For instance, when $r$ is greater than $1350 \mathrm{~kb} / \mathrm{s}$, two of the three users will be randomly picked to upload files, because more users can provide better services if they upload data with a greater rate.

In the final version of this paper, the authors plan to finish the simulation with more than three users. Moreover, the asymmetric case will be investigated and the distributed solution will be tested.

\section{CONCLUSIONS}

Selfish users in P2P file sharing always try to download files from the other users without contributing their own content. This may result in undesirable delay such as the bottleneck at the server. In order to improve the performance in file sharing, we propose a game-theoretic framework where users jointly optimize their strategies. With the correlated equilibrium concept, users are aware of the very long delay when there is little cooperation, so it is the best choice for them to jointly optimize their strategies and increase the degree of cooperation. Simulation results show that the delay is much smaller using the correlated optimal strategy than that of Nash equilibrium.

\section{REFERENCES}

[1] Napster. [Online]. Available: http://www.napster.com/

[2] The Gnutella Protocol Specification, 2000. [Online]. Available: http://dss. clip2.com/GnutellaProtocol04.pdf/

[3] Kazaa media desktop, 2001. [Online]. Available: http://www.kazaa.com/

[4] Bittorrent, 2003. [Online]. Available: bitconjurer.org/BitTorrent/

[5] E. K. Lua, J. Crowcroft, M. Pias, R. Sharma, and S. Lim, "A survey and comparison of peer-to-peer overlay network schemes," IEEE Communications Survey and Tutorial, Mar. 2004.

[6] S. Saroiu, P. Gummadi, and S. Gribble, "Measurement study of peer-topeer file sharing systems," Tech Report UW-CSE-01-06-02, University of Washington, 2001.

[7] B. Cohen, "Incentives build robustness in BitTorrent," 1st Workshop on Economics of Peer-to-Peer Systems, June 2003.

[8] S. Jun and M. Ahamad, "Incentives in BitTorrent induce free riding," in Proc. of the 2005 ACM SIGCOMM Workshop on Economics of Peer-toPeer Systems, 2005.

[9] D. Qiu and R. Srikant, "Modeling and performance analysis of BitTorrentlike peer-to-peer networks," in Proc. of ACM SIGCOMM, pp. 367-378, 2004.

[10] K. Lai, M. Feldman, I. Stoica, and J. Chuang. "Incentives for cooperation in peer-to-peer networks," in Workshop on Economics of Peer-to-Peer Systems, 2003.

[11] C. Buragohain, D. Agrawal, and S. Suri, "A game theoretic framework for incentives in P2P systems," in Proc. of the International Conference on Peer-to-Peer Computing, pp. 48-56, Sep. 2003.

[12] P. Golle, K. Leyton-Brown, and I. Mironov. "Incentives for sharing in peer-to-peer networks," ACM Electronic Commerce, Oct. 2001.

[13] F. Wu and L. Zhang, "Proportional response dynamics leads to market equilibrium," in Proc. of the 39th ACM symposium on Theory of computing, pp. 354-363, 2007.

[14] M. Osborne and A. Rubenstein, A Course in Game Thoery, MIT press, 1994.

[15] R. J. Aumann, "Subjectivity and correlation in randomized strategy," Journal of Mathematical Economics, vol. 1, no. 1, pp. 67-96, 1974.

[16] S. Hart and A. Mas-Colell, "A simple adaptive procedure leading to correlated equilibrium," Econometrica, vol. 68, no. 5, pp. 1127-1150, Sep. 2000.

[17] E. Altman, N. Bonneau, and M. Debbah, "Correlated equilibrium in access control for wireless communications," Lecture Notes in Computer Science, no. 3976, pp. 173-183, Springer-Verlag, Germany, 2006.

[18] A. Armengol, "The set of correlated equilibria of $2 \times 2$ Games," [Online] Available: http://selene.uab.es/acalvo/correlated.pdf.

[19] B. Shrestha, D. Niyato, Z. Han and E. Hossain, "Wireless Access in Vehicular Environments Using Bit Torrent and Bargaining", in Proceedings of IEEE Global Communications Conference, New Orleans, LA, November 2008.

[20] C. Papadimitriou, "Computing correlated equilibria in multiplayer games," [Online] http://www.cs.berkeley.edu/ christos/.

[21] L. Kleinrock, Queueing Systems, Wiley, 1975.

[22] Z. Han and K. J. R. Liu, Resource Allocation for Wireless Networks: Basics, Techniques, and Applications, Cambridge University Press, 2008.

[23] S. Boyd and L. Vandenberghe, Convex Optimization. Cambridge University Press, Cambridge, UK, 2004. 